\title{
Using Audiometry to Track Atherosclerosis: Measuring a Beneficial Effect of Methotrexate in Rheumatoid Arthritis
}

\author{
Authors: \\ Kylie Greenwald, *Maria Greenwald, JoAnn Ball \\ Desert Medical Advances, Palm Desert, California, USA \\ *Correspondence to dmaregulatory@gmail.com \\ Disclosure: $\quad$ The authors have declared no conflicts of interest. \\ Acknowledgements: Special acknowledgment to Gary Greenwald and Armando Garsd for their assistance \\ in editing and statistical analysis. \\ Received: $\quad 01.03 .20$ \\ Accepted: $\quad 07.04 .20$ \\ Keywords: $\quad$ Atherosclerosis, audiometry, methotrexate, rheumatoid arthritis. \\ Citation: $\quad$ EMJ Rheumatol. 2020;7[1]:110-117.
}

\section{Abstract}

Objectives: To correlate audiometry with atherosclerosis. Presbycusis is associated with age and atherosclerosis; a strong correlation might present opportunities to use audiometry to track atherosclerosis disease.

Design: The authors tested 87 elderly patients with rheumatoid arthritis (age range: 80-101 years; median: 86 years) with a history of methotrexate use for over 20 years. After 50 years of age, hearing loss begins slowly and by the age of 90 , the majority of the general population require hearing aids. In the 87 elderly participants, however, hearing was remarkably preserved.

Results: The observed cohort of 87 individuals showed better hearing than predicted compared to audiometry historically documented in the elderly $(p<0.001)$. The patients tested one to two decades younger than expected on audiometry and $44 \%$ of patients qualified for hearing aids instead of the expected $80 \%$, based on age.

Conclusion: The known reduction in atherosclerosis with methotrexate use in rheumatoid arthritis may account for this observed preservation of hearing., ${ }^{1,2}$ As hearing and atherosclerosis are related, the authors further postulated that routine audiometry may provide a cost-effective screening tool for other populations in future atherosclerosis studies.

\section{INTRODUCTION}

In the past 20 years, many studies have evaluated the relationship of systemic inflammation with atherosclerosis and coronary artery disease. ${ }^{3}$ Immune cells are prominent in early atherosclerotic lesions; cytokines further accelerate the arterial lesions, and inflammation activates clotting of platelets, all leading to myocardial infarction and stroke. Macrophages play a key role in arterial plaque and this macrophage activation can be modified by methotrexate. Hundreds of publications in the past decade have shown that treatment of the underlying inflammatory 
condition in rheumatoid arthritis significantly improved cardiovascular outcomes by reducing atherosclerotic disease. ${ }^{4-8}$ In a 25-year multicentre prospective study of 5,626 patients with rheumatoid arthritis, methotrexate was associated with a $70 \%$ reduction in mortality. Further prospective studies have demonstrated an additional $23 \%$ reduction of cardiovascular risk when methotrexate was utilised along with a biologic disease-modifying antirheumatic drug. ${ }^{9}$ Control of inflammation, therefore, reduced atherosclerotic disease. ${ }^{10}$

There are many known causes of hearing loss and the single strongest aetiology is atherosclerosis. Presbycusis, hearing loss, has been shown to be largely secondary to atherosclerotic vascular disease and is well described.11-13 Hearing acuity decreases with age and correlates strongly with the atherosclerotic small vessel disease in ageing. ${ }^{14}$ Audiometry measurement is objective, readily available, inexpensive, and reproducible. In most studies of atherosclerosis in clinical trials today, the primary outcome is a change in a radiologic measurement such as carotid intima media thickness over 5 years, and secondary outcomes include nonfatal myocardial infarction, nonfatal stroke, coronary artery bypass grafting, percutaneous coronary intervention, peripheral atherosclerotic arterial disease-related amputation, peripheral atherosclerotic arterial disease revascularisation, and death due to vascular disease. Unfortunately, these outcome measures require long duration and expenses, include end-of-life measures, and do not easily allow for a continuum of measurement for disease progression.

Using audiometry as a potential secondary outcome and surrogate measure to track small vessel atherosclerosis is a logical extension of the authors' understanding of the pathophysiology and offers an objective, repeatable low-cost measurement. Furthermore, it is not dependent on local culture or language. Clinical trials with interventions to prevent atherosclerosis could then potentially be monitored by audiometry in a continuous time course as a potentially useful secondary outcome.

This study evaluated a population of elderly patients with rheumatoid arthritis at high risk of atherosclerotic disease due to ongoing systemic inflammation. ${ }^{15}$ Rheumatoid arthritis populations have a 2-fold increased incidence of atherosclerosis compared to the normal population. Untreated rheumatoid arthritis is associated with increased mortality due to accelerated coronary artery and cerebrovascular atherosclerosis. The authors performed audiometry in a high-risk elderly population with rheumatoid arthritis (age range: 80-101 years old) who had been on continuous methotrexate for over 20 years. Significant presbycusis was expected in this group of individuals aged over 80 years old, but hearing was remarkably preserved so this prompted cross-sectional measurement of hearing in an observational study. For this group at high risk of atherosclerosis, because of rheumatoid arthritis with an underlying systemic inflammatory process, the authors hypothesised that that long-term methotrexate administration would be associated with both a low prevalence of atherosclerosis and a low level of hearing loss measured by audiometry. Rheumatoid arthritis does not cause hearing loss; the authors chose this cohort to study because rheumatoid arthritis has marked inflammation. Since chronic inflammation causes atherosclerosis and atherosclerosis causes hearing loss, treatment of chronic inflammation could result in less atherosclerosis and less hearing loss. In time, it followed that audiometry may prove to be a valuable, cost-effective marker to follow atherosclerosis.

\section{METHODS}

Conventional audiometry was performed on AMBCO 2500 equipment (AMBCO Electronics, Tustin, California, USA), in a single booth using the modified Hughson-Westlake procedure specified by the International Organization for Standardization (ISO) 8253-1:2010 Acoustics Audiometric test method. The same equipment was utilised on each subject and the testing was performed by the same technician. Results were recorded for each ear at 250, 500, 1,000, 2,000, 4,000, and 8,000 hertz $(\mathrm{Hz})$. Data was also analysed by quintile. Age, sex, language, socioeconomic status, education, medical history, tobacco use, concomitant medication, and vocation were recorded; additionally, all subjects had a physical and ear exam.

To evaluate proof of concept, this was a crosssectional observation trial. There were 87 
elderly individuals with rheumatoid arthritis enrolled sequentially, 15 were male and 72 were female. All subjects met the American College of Rheumatology (ACR) criteria for rheumatoid arthritis and were treated with methotrexate at time of disease onset. All subjects met the criteria for clinical remission, defined as Disease Activity Score-28 for Rheumatoid Arthritis with ESR (DAS28-ESR) <2.6, which accounts for solo methotrexate use for decades. Other activity measures such as Clinical Disease Activity Index (CDAI), Simple Disease Activity Index (SDAI), and Routine Assessment of Patient Index Data (RAPID) were not in use in the 1990s. Since these patients began methotrexate in the 1990s with good control of disease, other diseasemodifying antirheumatic drug use or biologic use in later years was a rare event. Participants were excluded if there was any history of trauma to the ear, vocations with known damage to hearing, prior damage from ear infection or recurrent sinus infection, allergic rhinitis requiring current therapy, Ménière's disease, chronic vertigo, chronic tinnitus, prior temporal-mandibular joint surgery, ototoxic medication use, or neurosurgery. There were three subjects who had hearing aids at baseline enrollment and the other 84 subjects stated they had never been advised to get a hearing aid nor felt a need for one. The need for hearing enhancement was based on reference criteria defined by the Ventry and Weinstein criteria ( $>40$ decibels $[\mathrm{dB}]$ hearing loss on the audiogram). ${ }^{16}$

All 87 individuals had been treated with methotrexate for over 20 years in remission and were sequentially requested to join the observational trial with audiometry. None of the individuals had cardiac disease, cerebral vascular disease, and all were able to climb a flight of stairs. No patient aged 80-101 was excluded from audiometry and ear examination. Patients were excluded if there was bone or conduction abnormality. Audiometry data collected to assess hearing loss was the average value of the two ear measurements to control for test-retest variance.

All participants signed consent for the audiometry and observational analysis as proposed in a protocol approved by the Western Institutional Review Board (WIRB). Results were provided free of charge to each participant with instructions to discuss the results with their primary physician. A full physical examination, ear examination, medical history, tobacco use history, and a list of concomitant medications was completed for each participant, none of whom were taking any corticosteroid medication.

\section{RESULTS}

Of the 87 sequentially enrolled patients, 15 were male and 72 were female. The ages ranged from 80 to 101, with men aged 80-96 and women aged 80-101. The average age among the men was 86.0 years (median: 86.0 years) and the average age among the women was 87.0 years (median: 85.5 years). Quintiles for audiometry exams were defined as normal (1 ${ }^{\text {st }}$ quintile [Q1]; 0-20 dB), mild impairment ( ${ }^{\text {nd }}$ quintile [Q2]; 20-4O dB), moderate impairment ( $3^{\text {rd }}$ quintile [Q3]; 40-60 $d B$ ), moderately severe impairment ( $4^{\text {th }}$ quintile [Q4]; 60-80 $\mathrm{dB}$ ), and severe impairment ( $5^{\text {th }}$ quintile [Q5]; 80-100 dB). Generally, a hearing aid was indicated for Q3, Q4, or Q5. Three male patients (20\%) had moderate or moderately severe impairment and one used a hearing aid. Thirty-six (50\%) of the elderly female patients had moderate to moderately severe impairment and two used a hearing aid. None of the participants had 'severe' hearing impairment (Q5) upon audiometry examination (Figure 1).

In Q1-Q5, there were 13\%, 67\%, 13\%, 7\%, and $0 \%$ male patients, respectively, and $16 \%, 34 \%$, $34 \%, 16 \%$, and $0 \%$ female patients, respectively. There was no statistical difference between males and females according to quintile. In the patient group, 44\% qualified for hearing aids $(n=39)$ although only three utilised any hearing enhancement. In analysis of the audiograms, there were no cases of otosclerosis (no Carhart notch), Ménière's disease, or evidence of trauma to the ear. No patient was enrolled if bone or conduction testing was abnormal. Duplicate measurement at the time of the audiogram showed hearing test results were reproducible with little variance.

There was an association between hearing loss and age in the group but this was substantially weaker than expected compared to large normative population studies (Figure 2 and 3).11,2,17-22 Expected hearing loss is $25 \%$ in individuals aged between 55 and 64 years, $43 \%$ in individuals aged between 65 and 84, and over $70 \%$ in individuals aged between 85 and 100 years old, as recorded by the Beaver Dam Offspring Study of ageing in 3,285 individuals. ${ }^{17}$ 


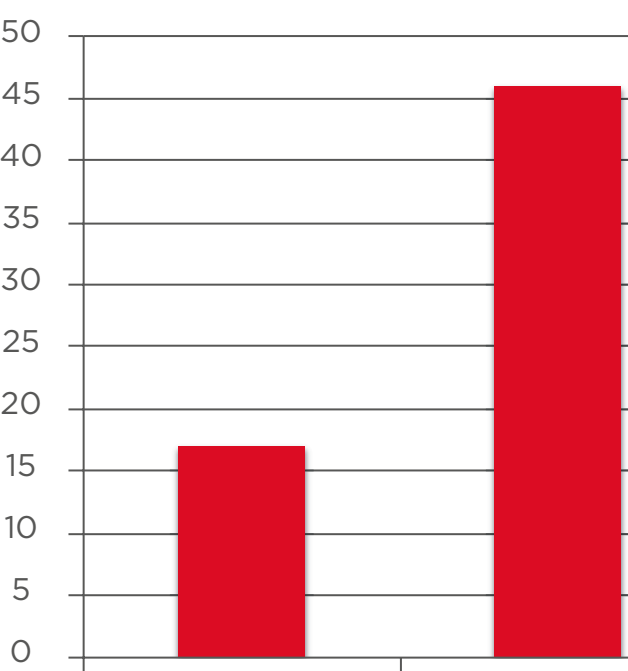

Q1
Q2

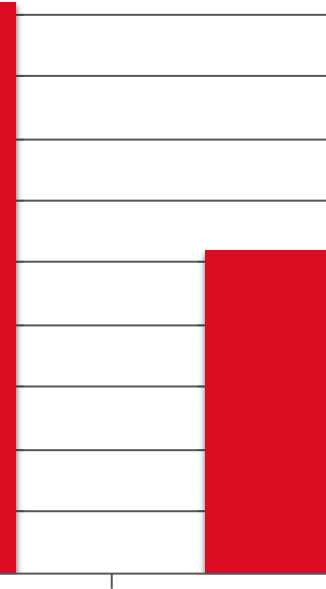

Q3

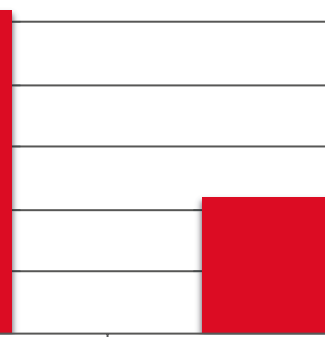

Q4

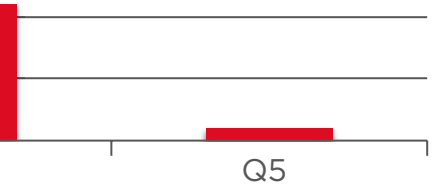

Q5

Quintile (\%)

Figure 1: First to fifth quintiles (total: 100\%).

Percentage of subjects in each quintile of hearing loss.
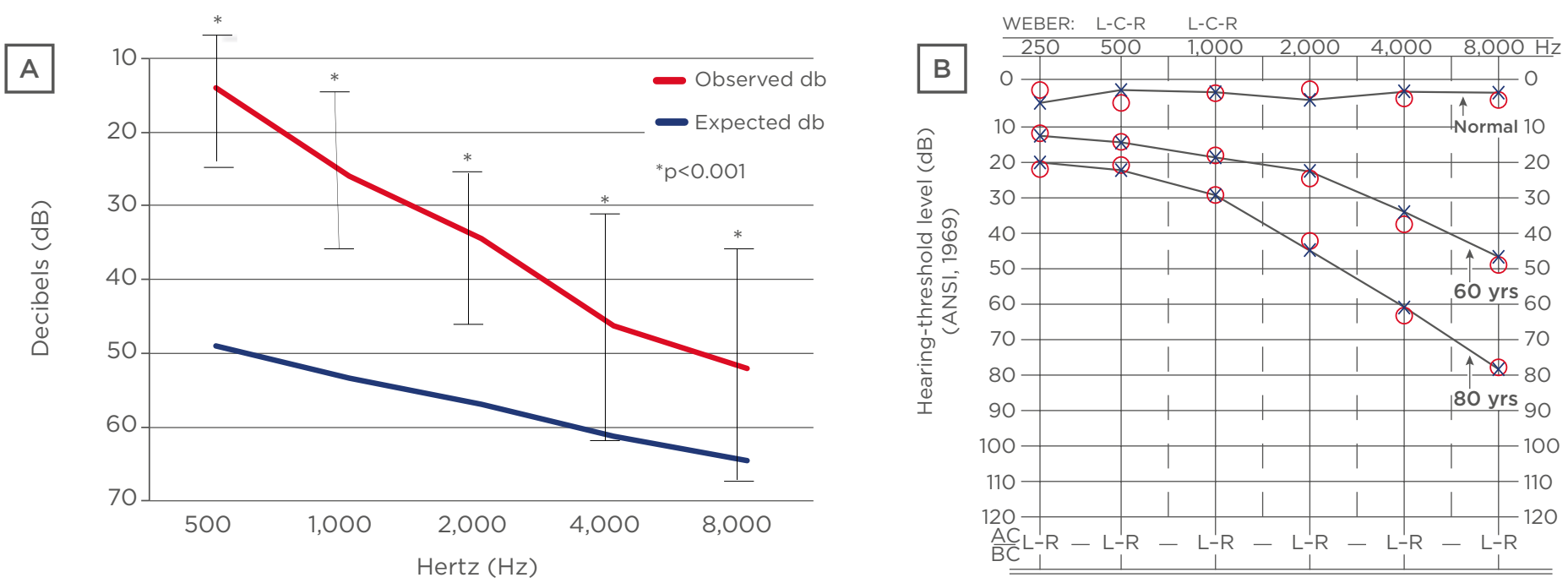

Figure 2: Pure tone audiometry.

(A) Observed cohort versus norms in the general population between 80-101 years old; (B) pure tone audiometry expected values in general population by age.

L: left; R: right; yrs: years.

Adapted from Blevins. ${ }^{20}$ 


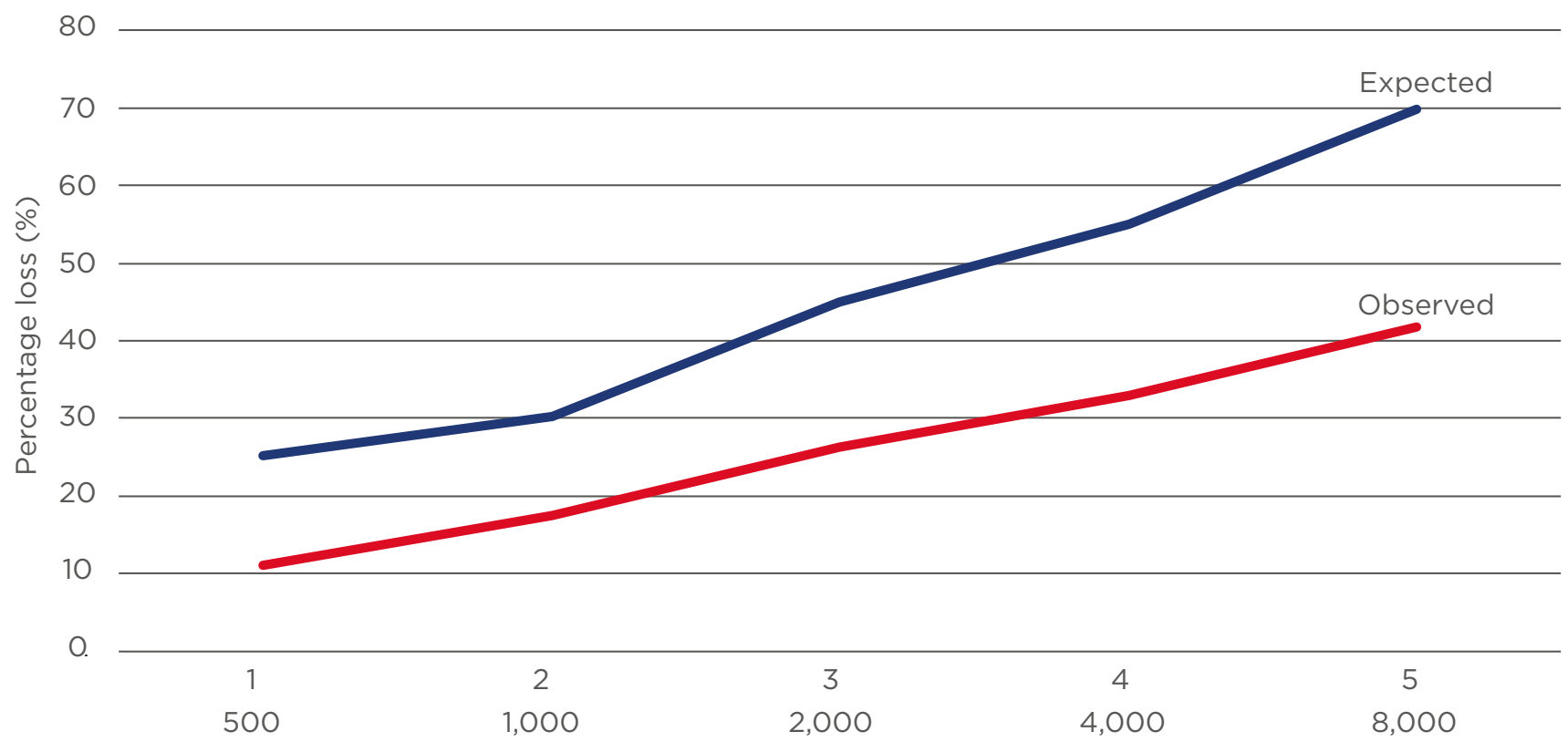

$\operatorname{Hertz}(\mathrm{Hz})$

Figure 3: Hearing loss above age 80.

Expected hearing loss with presbycusis and observed measurement of hearing loss in study cohort.

Surprisingly, more than half of the patients in this present study tested as normal or with only mildlyimpaired hearing despite advanced age (Q1 or Q2). Each measured audiogram frequency was statistically significantly different compared with preservation of hearing for the normal frequency in the patients aged 80-101 years old $(p<0.001)$. Results were not affected when adjusted for multiple variables including sex, tobacco use, statin use, language of origin, education, economic level, or vocation. The patients remained in clinical remission with an average DAS28-ESR 2.0 \pm 0.4 .

\section{DISCUSSION}

The U.S. Preventive Services Task Force (USPSTF) recommends that adults have an audiometry evaluation when they reach 50 years of age and every 3 years thereafter. ${ }^{23}$ Audiometry is important to every individual patient since corrections may be made, and presbycusis has a great impact on quality of life, self-esteem, depression, and isolation. ${ }^{24}$ The authors' hypothesis was that recognising hearing loss also marks increased risk for atherosclerosis, which may increase patient adherence to lifestyle changes, statins, and blood pressure control.

Normal hearing can range from 20 to $20,000 \mathrm{~Hz}$, but the range of frequencies for understanding speech is 500 to $4,000 \mathrm{~Hz}$. The standard objective test for hearing loss is the pure-tone audiogram, in which a patient is placed in a soundproof booth and tested on ability to hear tones at a series of discrete frequencies. Hearing loss begins with loss at high frequencies which carry the consonant sounds and thus the majority of speech information.

This study tested the $500-4,000 \mathrm{~Hz}$ range because it is clinically important and easy to obtain. The audiogram was repeated to obtain a mean of the measurements at each frequency to decrease test-retest error. Routine audiometry should be included in any good clinical practice for persons over 50 years of age, and the hypothesis raised by the observation of this study cohort may reflect early symptoms of atherosclerotic disease. If an audiometry test exists in every clinical chart for persons over 50 years of age, there may be data readily available to assess atherosclerosis risk for each patient. Although 
not used in this study, the AudioScope (Welch Allyn Inc., Skaneateles Falls, New York, USA) is a handheld screening instrument consisting of an otoscope with a built-in audiometer. It assesses the ability of patients to hear tones of 20,25 , and $40 \mathrm{~dB}$ at frequencies of 500, 1,000, 2,000, and $4,000 \mathrm{~Hz}$ and requires approximately 90 seconds to administer. ${ }^{23}$

This observational study attempted to evaluate if there was a correlation between protection against atherosclerosis and preservation of hearing. This was an observational study in the elderly (over 80 years of age), and evaluating audiometry as an atherosclerosis tool will require future studies comparing repeat audiometry measurements to current usage of CT scans, angiography, ultrasound, stress tests, and other radiographic imaging, as well as clinical outcomes such as myocardial infarction and stroke. Current ongoing clinical trials are in progress evaluating PET scan cardiology and concurrent audiometry in patients with high risk of atherosclerosis disease. ${ }^{25}$ The hope is that future trials treating atherosclerosis could collect information with audiometry being more cost-effective than repeat imaging, stress tests, or PET scans.

Hearing loss is well documented to progress linearly with age (Figure 2 ). ${ }^{20}$ Hearing amplification is generally indicated for hearing thresholds $>40$ $\mathrm{dB}$ on audiogram (Q3-Q5). Many very elderly patients, generally, would have a hearing aid recommended because most would fall into Q3Q5; in this study, it was found that $44 \%(n=39)$ would benefit from this ( $Q 3$ or Q4). Since most with thresholds $>40 \mathrm{~dB}$ were in $\mathrm{Q3}$, it is possible that people were managing without a hearing aid. It was an unexpected benefit of methotrexate in the patients that hearing was preserved, and most tested one to two decades younger than their age for expected hearing loss. A 100-year-old patient showed the expected results of an 80-year-old patient, and an 80-year-old patient showed the expected results of a 60-year-old patient on audiometry. The auditory technicians also noted preservation of hearing too when evaluating one of the patients on long-term methotrexate. This prompted the early motivation to collect the data in this observation project.

The under-utilisation of hearing aids in the general population was described by Wattamwar $\mathrm{K}$ et al." in 2017 in a study of 647 patients aged 80-106 years, where over $80 \%$ qualified for hearing aids, but only $59 \%$ used any hearing devices. ${ }^{11}$ Hearing aids are important when indicated: improved hearing ameliorates isolation, depression, irritating tinnitus, and emotional impact commonly associated with presbycusis. ${ }^{26}$

Hearing loss obviously has an adverse effect on quality of life daily but hearing loss may also reflect atherosclerotic disease resulting in death and cognitive decline. A prospective study by Gates et al. ${ }^{27}$ enrolled 274 patients in a surveillance programme of the general population and those at baseline with hearing loss had an increased risk for Alzheimer's disease or cerebral vascular disease over the 4-year follow-up. ${ }^{27}$ In another prospective study with 639 normal volunteers over 11 years, those with baseline hearing loss showed a significant increased incidence of cognitive decline, ${ }^{28,29}$ presumably due to increased small vessel cerebral disease. ${ }^{30,31}$ Hearing loss was a marker for atherosclerosis, both in coronary disease and cognitive function. ${ }^{32}$

The predominant cause for presbycusis is atherosclerosis, but other factors influence hearing over time such as oestrogen levels, traumatic harm to hair cells, cochlear processes, tobacco use, and statin use. ${ }^{17}$ Age, after accounting for other variables, accounts for approximately $10 \%$ of hearing decline. ${ }^{12}$ All 87 patients in this present study had been taking methotrexate for over 20 years and their rheumatoid joints were objectively in remission for many years. Long-term therapy in rheumatoid arthritis with methotrexate has correlated with significant cardiovascular benefit and decreased mortality. Based on published norms, the degree of hearing acuity preserved in this group of elderly patients was unexpected. Hundreds of studies published in the literature have shown methotrexate confers atherosclerotic benefits in rheumatoid arthritis. There are no prior published studies of audiometry in rheumatoid arthritis; rheumatoid arthritis does not cause presbycusis, but the atherosclerosis risk in rheumatoid arthritis may account for the presbycusis. If inflammation is controlled, this results in less atherosclerosis disease and less presbycusis.

Measurement of cognitive decline was minimal in patients from this current study with long-term use of methotrexate, elsewhere reported. ${ }^{33}$ It has been noted that hearing loss is linked to increased 
dementia and the authors speculated that the study group may have done well on cognitive testing partly because of both this preservation of hearing and decreased cerebral atherosclerosis. The preserved hearing acuity in the group contributed to the high cognitive scores. As atherosclerosis is a cause of presbycusis as well as small vessel vascular dementia, it is possible that interventions for preventing atherosclerosis may be followed using audiometry and cognitive testing beside the traditional outcome measures of myocardial infarction, stroke, and death. Certainly a major limitation of this observational study was that it was not a randomised prospective trial, and the cohort group could only be matched for age and sex, since the normal population cohort used for predicted hearing acuity did not have rheumatoid arthritis. Despite this disparity in the cohort groups, it was clear that the patients had far better hearing, presumably due to treatment of chronic inflammation leading to less atherosclerosis, so that future evaluation of atherosclerosis treatments may include audiometry as an inexpensive assessment.

Results of this population of patients with rheumatoid arthritis treated with methotrexate may not be extrapolated to the general population and this represents a limitation of the hypothesis. There is no comparison group of patients with rheumatoid arthritis after 20 years of disease without methotrexate, so the 'expected' hearing loss with age was drawn from the USA general population in epidemiologic studies for persons over the age of 80 . Furthermore, in studies evaluating the concept of inflammation leading to atherosclerosis, methotrexate was not found to suppress cardiovascular events in the general elderly population ${ }^{34}$ so there is no comparison elderly group with methotrexate. This study compared the measured audiometry results in the observational group of patients on methotrexate and compared hearing loss to the general population of the same age. In the future, audiometry might prove to be appropriate to assess atherosclerosis in the general population with other therapies such as statins, PCSK9 inhibitors, sodium glucose transport protein inhibitors, or other agents known to suppress inflammation. ${ }^{35}$ All of these therapies are approved to reduce atherosclerotic disease in the general population.

\section{CONCLUSION}

The authors hypothesised that audiometry as a continuous variable might identify individuals with increasing cardiovascular risk and may prove to be a valuable tool to assess therapy directed toward atherosclerosis. This was a proof-of-concept study in a population at high risk for atherosclerosis, and treated with methotrexate which has been shown to decrease atherosclerosis disease in rheumatoid arthritis. ${ }^{7}$ All patients had rheumatoid arthritis treated with long-term methotrexate to reduce atherosclerotic risk, as well as to control the inflammatory disease of arthritis and this study found preserved hearing. Other factors beyond atherosclerosis that co-vary with age contribute to presbycusis, but the authors speculate because of the high contribution of atherosclerosis to hearing loss, that repeat measurement of hearing may offer a useful, practical, and readily available surrogate measure for atherosclerosis progression.

\section{References}

1. Wasko M et al. Propensity-adjusted association of methotrexate with overall survival in rheumatoid arthritis. Arthritis and Rheumatism 2013;65(2):334-42.

2. Coomes $\mathrm{E}$ et al. Methotrexate in atherogenesis and cholesterol metabolism. Cholesterol. 2011;503028.

3. Hansson G. Inflammation, atherosclerosis, and coronary artery disease. N Engl J Med. 2005;352(16):1685-95.
4. Buch M et al. Treatment naive, early rheumatoid arthritis patients demonstrate reversible abnormalities of vascular function- a first, RCT derived longitudinal study. Abstract L05. Annual Meeting - American College of Rheumatology, 23 October, 2018.

5. Polachek A et al. Risk of cardiovascular morbidity in patients with arthritis: a meta-analysis of observational studies. Arthritis Care Res (Hoboken). 2017;69(1):67-74.

6. Roubille $\mathrm{C}$ et al. The effects of tumor necrosis factor inhibitors, methotrexate, non-steroidal anti-inflammatory drugs and corticosteroids on cardiovascular events in rheumatoid arthritis, psoriasis and psoriatic arthritis: a systematic review and meta-analysis. Ann Rheum Dis. 2015;74(3):480-9.

7. Naranjo A et al.; QUEST-RA Group. Cardiovascular disease in rheumatoid arthritis: QUEST-RA group. Arthritis Res Ther. 2008;10(2):R30.

8. Landewe R. Editorial: Methotrexate saves lives. Arth \& Rheum. 


\section{3:65(2):307-9.}

9. van Halm VP et al. Disease-modifying antirheumatic drugs are associated with a reduced risk for cardiovascular disease in patients with rheumatoid arthritis: a case control study. Arthritis Res Ther. 2006;8(5):R151.

10. Elnabawi $Y$ et al. Association of biologic therapy with coronary inflammation in patients with psoriasis as assessed by perivascular fat attenuation index. JAMA Cardiol. 2019;4(9):885-91. [Epub ahead of print].

11. Wattamwar $\mathrm{K}$ et al. Increases in the rate of age-related hearing loss in the older old. JAMA Otolaryngol Head Neck Surg. 2017;143(1):41-5.

12. Gates G, Cooper J. Incidence of hearing decline in the elderly. Acta Otolaryngol. 1991;111(2):240-8

13. Hull RH, Kerschen SR. Can improved cardiovascular health enhance auditory function? Hearing $\mathrm{J}$. 2018;71(2):22-3.

14. Livingston G, Sommerlad A, Orgeta V, et.al. Repeat audiograms predict atherosclerosis. Alzheimer Assn International Conference. Lancet. 2017;390:2673-2734.

15. Kaplan M. Cardiovascular disease in rheumatoid arthritis. Curr Opinion Rheumatol. 2006;18(3):289-97.

16. Weinstein B, Ventry M. Hearing impairment and social isolation in the elderly. J Speech Hear Res. 1982;25(4):593-9.

17. Nash SD et al. Prevalence of hearing impairment and associated risk factors: the Beaver Dam Offspring Study. Arch Otolaryngol Head Neck Surg. 2011;137(5): 432-9.

18. Stevens $\mathrm{G}$ et al. Global and regional hearing impairment prevalence: an analysis of 42 studies in 29 countries. Eur J Public Health. 2013;23(1):146-52

19. Houston D et al. Age-related hearing loss. Am J Clin Nutr. 1999;69(3):56471.

20. Blevins $\mathrm{NH}$. Presbycusis. 2020. Available at: https://www.uptodate. com/contents/presbycusis. Last accessed: 24 June 2020

21. Gopinath B et al. Prevalence of age related hearing loss in older adults: Blue Mountains study. Arch Intern Med. 2009;169(4):415-6.

22. Sprinzl G, Reichelmann H. Current trends in treating hearing loss in elderly people. Gerontology. 2010;56(3):351-8.

23. Agency for Health Care Research and Quality, U.S. Department of Health and Human Services. Screening for Hearing Loss in Adults Ages 50 Years and

Older: A Review of the Evidence for the U.S. Preventive

Services Task Force. 2011. Available at: https://www.ncbi.nlm.nih.gov/ books/NBK53864/pdf/Bookshelf NBK53864.pdf. Last accessed: 20 February 2020.

24. Simpson A et al. Time from hearing aid candidacy to hearing aid adoption: a longitudinal cohort study. Ear Hear. 2019;40(3):468-76.

25. National Heart, Lung, and Blood Institute (NHLBI). Links between inflammation and cardiometabolic diseases. NCTO1934660. https:// clinicaltrials.gov/ct2/show/ NCT01934660.

26. Nash SD et al. Unmet hearing health care needs: the Beaver Dam Offspring Study. Am J Public Health. 2013;103(6):1134-9.
27. Gates GA et al. Central auditory dysfunction as a harbinger of Alzheimer dementia. Arch Otolaryngeal Head Neck Surg 2011;137(4):390.

28. Lin FR et al. Hearing loss and cognition among older adults in the United States. Neuropsychol. 2011;25(6):763-70.

29. Lin $\mathrm{F}$ et al. Hearing loss and dementia. Arch Neurol. 2011;68(2):214-20.

30. Gallacher J et al. Auditory threshold, phonologic demand and incident dementia. Neurology. 2012;79(15):1583-90.

31. Deal $\mathrm{J}$ et al. Hearing impairment and incident dementia and cognitive decline in older adults: the Health ABC Study. J Gerontol A Biol Sci Med Sci. 2017;72(5):703-9.

32. Satizabal $\mathrm{CL}$ et al. Incidence of dementia over Three Decades in the Framingham Heart Study. N Engl J Med. 2016;374(6):523-32.

33. Greenwald $\mathrm{K}$ et al. ABO247 Lack of ageing with long term methotrexate: objective measurements of cognition, audiometry, and sleep. Ann Rheum Dis. 2018;77:1305.

34. Ridker P et al.; CIRT Investigators. Low-dose methotrexate for the prevention of atherosclerotic events. N Engl J Med. 2019;380:752-62.

35. Ridker $P$ et al.; CANTOS Trial Group. Antiinflammatory therapy with canakinumab for atherosclerotic disease. N Engl J Med. 2017;377:1119 31. 